\title{
A mechanism for encouraging active consumers to optimize the operations of power supply systems
}

\author{
Natalia Aizenberg ${ }^{1,}$, Nicolai Voropai $^{1}$, and Elena Stashkevich $^{2}$ \\ ${ }^{1}$ Melentiev Energy Systems Institute of SB RAS, Irkutsk, Russia \\ ${ }^{2}$ National Research Irkutsk State Technical University, Irkutsk, Russia
}

\begin{abstract}
We propose to analyze an interaction of power supply company and consumers as a game in which the retailer's goal is to maximize its profit, and the consumer's goal is to maximize its utility. The utility function of each consumer is described proceeding from the form of the loss function of the consumer, and one of our goals in the submitted paper is to find how to describe this load function mathematically. We want to develop an economic mechanism that help the power retailer to find an optimal strategy on the set of possible loads and tariffs. They should satisfy a set of constraints binding these two variables by natural requirements imposed on the values of the consumer utility functions.
\end{abstract}

\section{Introduction}

Consideration is given to the interaction among electricity consumers and an electric power retailer in which each consumer tries to reduce the cost of electricity by redistributing (shifting) its demand (or load) between peak hours and low-demanded ones, whereas the power retailer tries to reduce its expenses associated with storing electricity, especially, at peak hours $[1,2]$. We propose to analyze such an interaction as a game in which the retailer's goal is to maximize its profit, and the consumer's goal is to maximize its utility. The utility function of each consumer is described proceeding from the form of the loss function of the consumer, and one of our goals in the submitted paper consists of finding how to describe this load function mathematically. However, the major goal is to develop an economic mechanism that would help the power retailer (considered as a monopoly in the marketplace) maximize its profit based upon the known loads of all the consumers. We consider a finite number of different types of the so-called "active" (load-controlled) consumers based upon their incentives to optimize their loads. Each type of the incentives (and, consequently, consumers) is defined by a utility function of the consumer, which is the function of the hour load volumes within a 24-hour segment. We offer an alternative utility function to describe the behaviour of a consumer who is not an "active" one (since this consumer does not optimize the consumption of electricity by means of shifting its loads from the peak hours to low-cost ones). We formulate the problem of finding an optimal strategy for the power retailer as the one of maximizing the profit that the retailer can make with respect to each type of the "active" consumers on the set of possible loads and tariffs that satisfy a set of constraints binding these two variables by natural requirements imposed on the values of the consumer utility functions.

The use of market mechanisms and development of information and communication technologies encourage active behaviour of another participant of the electricity supply process, i.e. power supply company, as well. The company becomes capable of encouraging consumers to actively manage their electricity consumption [3-8] by establishing price options in real time. The interaction between the power supply company and consumers requires coordination of their operating conditions. Until now the potentialities of the coordination have been studied on the basis of mathematical programming methods, including two-stage stochastic programming $[8,9]$, a multi-agent $[10-14]$ and a game-theoretic $[9,10$, 12-14] approaches.

The behaviour and strategies of load-controlled consumers have been studied by many researchers in the field. The authors of [15] study one such problem in which for any consumer of the power retailer of electricity, a disclosure of any information on its load is not mandatory is considered, and the power retailer tries to offer stimulating tariffs to all the consumers while even determining sources of saving for the consumers. Situations in which the prices for electricity are determined in advance so that each consumer actively changes the load in order to reduce its expenses as much as possible are considered in $[12,13]$. Variants of a twolevel formulation of the problem in which the interaction in considered for a set of vertically integrated legal entities such as a generating company, a retailer, a network and a consumer, which is represented in an aggregate form, without division into types, are presented in [5]. In some statements of the problem, consumers are allowed to supply any electricity surplus

Corresponding author: ayzenberg.nata@gmail.com 
back to the network [16]. There are statements dealing with an additional generation of electricity, when consumers can only shift load from the peak time via changing their production process, in particular, by using electricity storage systems or by automating the production process [4].

The subject of consideration in the present paper differs from those in the enumerated studies. That is, we consider consumers of several types each having different incentives to optimize their load, and we show how to determine the tariffs harmonized for both fully rational and bounded rational consumers. Here, we consider a customer to be fully rational if it does not vary its load, whereas we consider a customer to be bounded rational if it may change its load curve by shifting part of its load during peak hours to low-demand ones. The latter is offered a tariff stimulating load optimization. Standard solution to the problem of profit maximization of an electricity supply organization under the presence of asymmetric information on the agent type can lead to the formation of conditions for the adverse selection. We form constraints to determine the contracts (tariffs) that make it possible to search for a separating equilibrium where each type of consumers selects "their" tariff and adjusts their consumption as is necessary for the system. The research formulates the incentive rationality constraints and incentive compatibility constraints for the time-of-use and two-rate tariffs.

\section{The Model}

There are a finite number of different types of the socalled "active" (load-controlled) consumers who have the ability to adjust own load, and consumer who does not optimize the electricity consumption by means of shifting its loads. $\Theta$ is the set of $N$ types of consumers $\theta \in \Theta$. There are $K$ consumers in model. Each consumer derives some utility from receiving a certain amount of electricity. Denote this utility through the function $u_{k}\left(q_{t k}^{\theta}\right)$ where the load at a certain $t$-zone of the day is $q_{t k}^{\theta}, q_{t k}^{\theta} \geq 0 . R_{t}^{\theta}$ is a tariff value at hour $t$, $t \in[1, T]$ for type $\theta$. The problem of each $k$ consumer is the maximizing of gains from electricity buying with respect to $q_{t k}^{\theta}, k=\overline{1, K}$ :

$$
\begin{aligned}
& U^{\theta}(\theta, q)=\sum_{t=1}^{T} u_{k}\left(q_{k t}^{\theta}\right)-R^{\theta} \rightarrow \max _{q}, \\
& q_{k t}^{\theta} \geq 0, \quad k \in \overline{1, K}, \quad \theta \in \Theta .
\end{aligned}
$$

The function of utility usually is monotonically increasing and concave. It should be performed additional property of Spence-Mirrlees:

$$
\frac{d U^{\theta}(\theta, q)}{d q} \geq 0, \frac{d^{2} U^{\theta}(\theta, q)}{(d q)^{2}} \leq 0, \frac{d^{2} U^{\theta}(\theta, q)}{d \theta d q} \geq 0 .
$$

The number of $\theta$-type consumer is $m^{\theta}$ and $\sum_{\theta \in \Theta} m^{\theta}=K$. The power supply company appoints the tariff for $\theta$-type of consumer $R_{t}^{\theta}$ using the total load $\sum_{k=1}^{m^{\ominus}} q_{k t}^{\theta}$ and its cost function $C\left(\sum_{k=1}^{m^{\theta}} q_{k t}^{\theta}\right)$. The cost function is convex with respect to $\sum_{k=1}^{m^{\theta}} q_{k t}^{\theta}$. The main purpose of the power supply company is to maximize profits of tariffs $R_{t}^{\theta}$ :

$$
\begin{aligned}
& \pi=\sum_{\theta \in \Theta}\left(R^{\theta}-\sum_{t=1}^{T} C\left(\sum_{k=1}^{m^{\theta}} q_{k t}^{\theta}\right)\right) \rightarrow \max _{R^{\ominus}}, \\
& R^{\theta} \geq 0 .
\end{aligned}
$$

We can decide the problem of select the tariff menu provided optimization the load curve through the coordination of interests of all stakeholders (1) and (2). We will update the form of utility functions and rewrite a problem through the theory of contracts.

The problem (1) is rewritten in terms of the conditions of participation and of individual rationality constraints for consumer types, which mean that the selection of "their" tariff proves more beneficial than the selection of a tariff of another consumer type. Moreover, we will assume that there is always some basic tariff which determines some alternative utility of $k$-th consumer $-\bar{U}_{k}$. This alternative utility will be derived by a consumer without participating in the selection of an optimal consumption pattern.

Then the general problem can be formulated as follows.

$$
\left\{\begin{array}{l}
\pi=\sum_{\theta \in \Theta}\left(R^{\theta}-\sum_{t=1}^{T} C\left(\sum_{k=1}^{m^{\theta}} q_{k t}^{\theta}\right)\right) \rightarrow \max _{R^{\theta} q_{k t}^{\ominus}} ; \\
\sum_{t=1}^{T} u_{k}\left(q_{k t}^{\theta}\right)-R^{\theta} \geq \bar{U}_{k}, \quad k \in\left[1, m^{\theta}\right], \theta \in \Theta ; \\
\sum_{t=1}^{T} u_{k}\left(q_{k t}^{\theta}\right)-R^{\theta} \geq \sum_{t=1}^{T} u_{k}^{\theta}\left(q_{k t}^{\circ}\right)-R^{\circ}, \quad k, j \in[1, K] ; \\
\forall \theta, \mathrm{o} \in \Theta, q_{k t}^{\theta} \geq 0, q_{k t}^{\circ} \geq 0 ; R^{\theta} \geq 0, R^{\circ} \geq 0 .
\end{array}\right.
$$

The variables are the load of the individual consumer $q_{t k}^{\theta}$ and the tariffs value $R_{t}^{\theta}$ which is offered by the power supply company. We consider the example of the two types of consumer: with full rationality and with bounded rationality. Let describe utility function of $\theta$ type consumers.

\subsection{The determination of optimal tariff for the two types of consumers}

I. At first the problem of utility maximization for the participation of the rational consumer $\theta_{1} \in \Theta$ is described. The constraints that include the conditions of a positive utility in selecting the tariff are the conditions of participation. In our research we believe that such a 
consumer can use a two-part tariff. In this tariff is assumed that the electricity unit price corresponds to the marginal cost of a electricity unit, and fixed costs are covered through an entrance fee (or fee peak power). Should take into account that with such scheme due to the absence of constraints the monopolist can confiscate the full consumer surplus, the value of which may exceed the fixed costs of power supply company. The government should regulate the entrance fee.

The utility function of the consumer with the full rationality is written via consumer's loss function or as the tariff $U^{\theta_{1}}=-R^{\theta_{1}}$ of $\theta_{1}$-type offered by the power supply company. $R^{\theta_{1}}$ is two-part tariff.

$$
R^{\theta_{1}}=A_{\theta_{1}}^{\text {peak }}+\sum_{t=1}^{24} T_{t}^{\theta_{1}} q_{\mathrm{t}}^{\theta_{1}}
$$

where $A_{\theta_{1}}^{\text {peak }}$ is entrance fee or the price of the maximum power, $T_{t}^{\theta_{1}}$ is the tariff value at hour $t, t \in[1,24]$. If the consumer selects the tariff $R^{\theta_{1}}$ and optimize the load, then she wants the expenses less than the costs for her consumption unchanged. Then the condition for the consumer's participation with full rationality is $-R^{\theta_{1}} \geq \bar{U}$.

The power supply company use the possibility of withdrawal the consumer surplus as much as possible. Therefore, we get that power supply company increases the tariff (under the entrance fee) until the condition of participation allows. Thus the consumer load does not change because of her rationality.

It means that condition of participation for nonactive participant fulfils as equality in the problem (3).

II. Now let us write the utility function for consumer with bounded rationality. The costs of this consumer are defined via differentiated tariff. Let $T_{j}^{\theta_{2}}$ is the tariff of $j$ th time zone, $j \in\{I, I I, I I I\}$.Then the differentiated tariff is

$$
R^{\theta_{2}}=T_{I} \cdot \sum_{j \in I} q_{j}^{\theta_{2}}+T_{I I} \cdot \sum_{j \in I I} q_{j}^{\theta_{2}}+T_{I I I} \cdot \sum_{j \in I I I} q_{j}^{\theta_{2}} .
$$

Let's formulate the problem of maximizing the consumer profits when the load shifts from a time zone to another one. The retail price in the spot market is $P_{t}^{S}$, where $t$ defines the purchase zone; $P_{t}^{B}$ is the price in the balancing market; $x$ is a potential scope of load optimizing by consumer (here we consider the scope of load shift from the peak time to the night time), $x \geq 0$. Then the payoff of the power supply company from the load shift can be written in the form

$$
S(\delta, x)=\left(P_{I I I}^{B}-P_{I}^{S}\right) \cdot x-\delta \cdot x,
$$

where $\delta$ is step of tariff $T_{t}$ which are established by the retail company at different hours of the day. For simplicity we can assume that $\delta=T_{I}-T_{I I I}$. Here we represent a cost function of power shortage in a simplified form. The function takes into account only the price difference in the balancing and spot markets. There can be additional costs not related to the price difference, to eliminate the shortage.

We assume that the consumer costs related to the load shift is the function of the load $f(x), f(x) \in C^{2}$. Here, we suppose that some load will be shifted by consumer without any special efforts. For example, this may concern the use of washing and dishwashing machines. At the same time, there is a load whose shift can cause greater inconveniences, for example, a shift of the food cooking time or additional installation of energy saving equipment. The function $f(x)$ reflects an extent to which the consumer is ready to such costs. The total payoff of the consumer will be represented as

$$
\begin{aligned}
U^{\theta_{2}}=- & \left(T_{I I I}+\delta\right) \cdot\left(\sum_{j \in I} q_{j}^{\theta_{2}}-x\right)-T_{I I} \cdot \sum_{j \in I I} q_{j}^{\theta_{2}}- \\
& -T_{I I I} \cdot\left(\sum_{j \in I I I} q_{j}^{\theta_{2}}+x\right)-f(x) .
\end{aligned}
$$

Consumer determines the load to be shifted to maximize her payoff $U^{\theta_{2}}$. For example

$$
f(x)=-\beta \cdot \log (1-x / \alpha) .
$$

Each parameter can be interpreted in the following way. Parameter $\alpha$ (asymptote) is a maximum possible load that can be shifted by the consumer from the peak time. Parameter $\beta$ controls the level of costs expressed in some money equivalent. Here this parameter is estimated on the basis of empiric data on the possibilities of load change for the considered categories of consumers. Let us take for an example the function which can describe potential consumer expenses caused by the load shift. The measurement units can be represented by money units, but here we assume that these units are an equivalent of the consumer efforts to change her load curves. The base consumer problem is to maximize the utility:

$$
U^{\theta_{2}} \rightarrow \max _{x}
$$

The solution is $\delta=f^{\prime}(x)$. Let the function of costs for power supply company be a concave function of $x$. To find the Nash equilibrium and to match the interests of all the stakeholders, it is necessary to find a point such that the maximum profit for the power supply company and the maximum payoff for the consumer are reached at the same time.

$$
\begin{gathered}
S(\delta, x) \rightarrow \max _{\delta} ; \\
\delta=f^{\prime}(x), x \geq 0, \delta \geq 0 .
\end{gathered}
$$

The conditions mean that the consumer will shift a certain load at different tariff difference $\delta$ :

$$
\left(P_{I}^{B}-P_{I I}^{S}\right) \cdot\left(f^{\prime}\right)^{-1}(\delta)-\delta \cdot\left(f^{\prime}\right)^{-1}(\delta) \rightarrow \max _{\delta}, \delta \geq 0 .
$$


Using (4) we have that $\delta=\sqrt{\beta\left(P_{I}^{B}-P_{I I}^{S}\right)}$.

\subsection{Solving the problem on the differentiation of consumer types and determination of an optimal tariff}

Let an alternative utility is determined by means a basic linear (i.e., constant in each time of the day) tariff $T^{L}$. We want that the consumer of rationality type will prefer two-part tariff, while the consumer of bounded rationality type will prefer differentiated tariff. Let us write the conditions of participation.

For the consumer of full rationality type:

$$
U^{\theta_{1}} \geq-T^{L} \sum_{t=1}^{24} q_{t}^{\theta_{1}}
$$

For the consumer of bounded rationality type:

$$
U^{\theta_{2}} \geq-T^{L} \sum_{t=1}^{24} q_{t}^{\theta_{2}}
$$

We already know that restriction (5) in the problem (3) is active for the consumer of full rationality type. The individual rationality constraints for the consumer of this type means that the choice of this tariff is more beneficial for the consumer, than choosing another types of consumer tariff.

$$
\begin{aligned}
& U^{\theta_{1}} \geq-\sum_{t \in\{I, I, I I I\}} T_{t} \cdot q_{t}^{\theta_{1}}, \\
& U^{\theta_{2}} \geq-\left(A_{\theta_{2}}^{\text {peak }}+\sum_{t=1}^{24} T_{t} q_{\mathrm{t}}^{\theta_{2}}\right) .
\end{aligned}
$$

It's easy to prove by the methods of the contract theory that the restriction (7) is active. Here, we do not consider the case, when consumers with bounded rationality choose a two-part tariff, and at the same time shift their loads, since the statement of the problem in this situation changes. Our objective is just to encourage consumers with bounded rationality to optimize their load curves. If any consumer can optimize its load curve without an additional action from the power supply company, it refers to the rational type $\theta_{1}$.

The function of the power supply company profit is concave.

$$
R^{\theta_{1}}+R^{\theta_{2}}-C\left(q^{\theta_{1}}, q^{\theta_{2}}\right)+S(\delta, x) \rightarrow \max _{\delta, x, R^{\theta_{1}}}
$$

where $C\left(q^{\theta_{1}}, q^{\theta_{2}}\right)$ is the total cost function of the power supply company for power purchase at the market and its delivery to a consumer. $S(\eta, x)$ is the profitability from the possible shift to another time zone. The variables are the step values in the differentiated tariff and prices in the two-part tariff. Restrictions (5) and (8) are active, (7) run as strict inequalities, (6) is superfluous.

By solving the problem of power supply company profit maximization (8) subject to constraints (4)-(7), we get the equilibrium, i.e., the optimal tariffs. These tariffs match the interests of different types of consumers and suppliers.

\section{Example: the search of optimal power supply company strategy in the case of two types of consumers}

The several types of consumers is considered in the example of the campus. The set of consumers forms the load curve of a power system. The consumers are hostels, educational buildings, student's catering facilities, municipal health centre and prophylactic sanatorium.

We consider the health centre and prophylactic sanatorium as the unique consumer of rationality type, consumer $\theta_{1}$, her load is not changed. She should choose a two-rate tariff $R^{\theta_{1}}$ with large payment for connection (power) and non-differentiated small payment per unit of electricity. Another group are consumers with bounded rationality, their loads are changed: $\theta_{2}^{1}$ for hostels, educational buildings and students' catering facilities; and $\left(\theta_{2}^{2}\right)$ for cafe). They should choose a differentiated tariff $R^{\theta_{2}}$ per electricity unit at a zero rate for power.

Table 1. The results of the interaction of the power supply company and three customers of power tariffs established by the state.

\begin{tabular}{|c|c|c|c|}
\hline $\begin{array}{c}\text { Consumer spending, } \\
\text { rub/day }\end{array}$ & $\theta_{2}^{1}$ & $\theta_{2}^{2}$ & $\theta_{1}$ \\
\hline$R^{\theta_{1}}$ & $\mathbf{2 9 7 4 4 . 4}$ & $\mathbf{1 8 8 4 7 . 8}$ & $\mathbf{1 3 0 2 7 . 5}$ \\
\hline$R^{\theta_{2}}$ & 32418.9 & 19202.2 & 15326.7 \\
\hline $\begin{array}{c}\text { Shortage of power, } \\
\text { kWt }\end{array}$ & 1065 \\
\hline $\begin{array}{c}\text { Company's income, } \\
\text { rub/day }\end{array}$ & $\mathbf{8 7 6 7 , 5}$ \\
\hline
\end{tabular}

Table 1 represents the results of the application of tariffs imposed by the state (see [17], the starting point). These results define the company's income, the consumer spending and the possible shortage of power. All consumers choose identical tariff (indicated in bold), this is a situation of adverse selection with high shortage.

Table 2. The results of the interaction of the power supply company and three customers after optimization of tariffs.

\begin{tabular}{|c|c|c|c|}
\hline $\begin{array}{c}\text { Consumer spending, } \\
\text { rub/day }\end{array}$ & $\theta_{2}^{1}$ & $\theta_{2}^{2}$ & $\theta_{1}$ \\
\hline$R^{\theta_{1}}$ & 29744,4 & 16777,4 & $\mathbf{1 3 0 2 7 , 5}$ \\
\hline$R^{\theta_{2}}$ & $\mathbf{2 8 4 7 6 , 2}$ & $\mathbf{1 6 3 6 1 , 2}$ & 14265,7 \\
\hline $\begin{array}{c}\text { Shortage of power, } \\
\text { kWt }\end{array}$ & 340 \\
\hline $\begin{array}{c}\text { Company's income, } \\
\text { rub/day }\end{array}$ & $\mathbf{1 0 0 9 0 . 3}$ \\
\hline
\end{tabular}

Further, we are determined the type of consumers that can be interested in choosing "not their tariff", then we solve the main problem (8) (see table 2). This way we 
find the conditions for the mixed equilibrium and for the equilibrium differentiating the types.

\section{Example: the search of optimal power supply company strategy in the case of two types of consumers}

The proposed methodology of the tariff determination allows both categories of consumers interacting with a power retailer - fully rational (who do not vary their load) and bounded rational (who change their load curve by shifting part of peak load) - to optimize the electricity supply to them under tariffs stimulating their load optimization. The profit maximization problem to be solved by the power retailer under the presence of asymmetric information on the consumer type is formulated in the paper in such a manner that its set of constraints contains the incentive rationality constraints and those of incentive compatibility. This allows consumers of each type to select "their" tariffs and adjust their consumption as it is necessary for the system as a whole.

For an industrial area (the city of Irkutsk), different consumer types have been identified, and utility functions for all the consumer types have been proposed. The choice of these proposed functions has helped implement the system of stimuli for the load optimization (in the sense of shifting the load from peak hours to off-peak ones) by all the consumers with bounded rationality of their preferences. The equilibrium tariffs have been calculated, whereas the tariffs approved by the legislature have been tested. The (tested) inefficiency of the latter follows, in particular, from their unprofitability for the power retailer. Indeed, since as long as part of load is not shifted from peak hours to off-peak ones, the shortage of electricity becomes inevitable, and the retailer expenses associated with shorting electricity increase substantially. In contrast, the tariffs calculated on the basis of the proposed approach can make the abovementioned system of stimuli for optimizing the consumer loads working, which reduces the probability of emerging shortages in electricity supply.

This work was supported Russian Foundation for Basic Research, № 16-06-00071.

\section{References}

1. N.I. Voropai, Z.A. Styczynski, E.V. Kozlova, V.S. Stepanov, K.V. Suslov, Proceedings of the Russian Academy of Sciences. Power engineering, 1, 84-90 (2014)

2. N. Aizenberg, E. Stashkevich, N. Voropai, Proceedings of the Russian Academy of Sciences. Power engineering, 3, 15-25 (2016)

3. G.G. Grebenyuk, M.M. Soloviev, Remote Control, 5, 166-173, (2004)

4. I.O. Volkova, M.V. Gubko, E.A. Salnikova, Control Sciences, 6, 53-61 (2013)

5. E. Pettersen, A.B. Philpott, S.W. Wallace, Decision support systems, 40(3), 427-438 (2005)

6. N. Aizenberg, E. Stashkevich, Proc. Int. Conf. on Problems of Critical Infrastructures, 25-27 (2015)

7. K. Sharma, C. Bhattacharya, IEEE Trans. on Smart Grid, 6, 2, 795-807 (2015)

8. R. Shigenove, A. Yona, T. Senjyu, Proc. ICEE (2015)

9. N. Good, E. Karangelos, A. Navarro-Espinosa, P. Mancarella, IEEE Trans. on Smart Grid, 6(3), 23332342 (2015)

10. R. Olfati-Saber, J. Fax, Proceedings of the IEEE, 95(1), 215-233 (2007)

11. M.R. Sheikhi, Sh. Bahrami, A.M. Ranjbar, IEEE Trans. on Smart Grid, 6(2), 675-683 (2015)

12. A.H. Mohsenian-Rad, V.W. Wong, J. Jatskevich, R.Schober, A. Leon-Garcia, IEEE Trans. on Smart Grid, 1(3), 320-331 (2010)

13. H. Chen, Y. Li, R.H. Louie, B. Vucetic, Autonomous IEEE Trans. on Smart Grid, 5(4), 1744-1754 (2014)

14. J. Chai, Z. Chen, Y. Yang, Zhang, IEEE Trans. on Smart Grid, 5(2), 1340-1350 (2014)

15. Z.M. Fadlullah, D.M. Quan, N. Kato, I. Stojmenovic, IEEE Systems journal, 8(2) (2014)

16. V.S. Stepanov, K.V. Suslov, E.V. Kozlova, Prom. Energetika, 6, 2-7 (2013)

17. Resolution of the Government of the RU of 2012 no.442.http://www.sbyt.irkutskenergo.ru/qa/2103.ht $\underline{\mathrm{ml}}$ 